Pacific Journal of Mathematic 


\section{AN ALGEBRAIC CRITERION FOR IMMERSION}

\section{BARRETT O'NeILL}

Let $R$ be the curvature tensor of a simply connected $d$-dimensional $(d \geq 4)$ Riemannian manifold $M$. T. Y. Thomas [2] has proved that if the rank of $R$ is not too small, there exist conditions expressed in terms of polynomials in the coordinates of $R$ which are satisfied if and only if $M$ can be immersed in the Euclidean space $R^{d+1}$. The proof is existential; the polynomials are not all given explicitly. Using the notion of Grassmann algebra we shall find a single, rather simple condition on $R$ necessary and sufficient for the existence of an immersion $i: M \rightarrow \bar{M}(K)$ with second fundamental form of rank at least four, where $\bar{M}(K)$ is a complete $(d+1)$-dimensional Riemannian manifold of constant curvature $K$. If coordinates are introduced this condition can be expressed algebraically in terms of polynomial equations and inequalities in the coordinates of $R$. The case $K=0$ yields an explicit variant of Thomas' result.

1. A differential criterion for immersion. Following [1] we fix the following notation for the structural elements associated with a $d$ dimensional $C^{\infty}$ Riemannian manifold $M: F(M)$, the bundle of frames on $M: R_{a}$, right-multiplication of $F(M)$ by $a \in O(d)$, the group of $d \times d$ orthogonal matrices; $\varphi$, the 1 -form of the Riemannian connection. Thus $\varphi=\left(\varphi_{i j}\right)$ is a vertical equivariant 1 -form on $F(M)$ with values in the Lie algebra of $d \times d$ skew-symmetric matrices. (We assume throughout that $1 \leq i, j, k \leq d$.$) Let \omega=\left(\omega_{i}\right)$ be the usual horizontal equivariant $R^{d}$-valued 1 -form on $F(M)$ defined by $\omega_{i}(x)=\left\langle d \pi(x), f_{i}\right\rangle$, where $x$ is in the tangent space $F(M)_{f}$ to $F(M)$ at $f=\left(f_{1}, \cdots, f_{a}\right)$ and $\pi$ is the natural projection. The curvature form $\Phi=\left(\Phi_{i j}\right)$ is by definition $D \phi$, the horizontal part of $d \varphi$. In the case of 1 -forms or 1 -vectors we write $x y$, rather than $x \wedge y$, for the Grassmann product.

Theorem 1. Let $M$ be a simply connected d-dimensional Riemannian manifold, $\bar{M}$ a complete $(d+1)$-dimensional Riemannian manifold of constant curvature $K$. Then $M$ can be immersed in $\bar{M}$ if and only if there exists a horizontal equivariant $R^{a}$-valued 1 -form $\sigma=\left(\sigma_{i}\right)$ on $F(M)$ such that

$$
\begin{cases}\Sigma_{k} \sigma_{k} \omega_{k}=0 & \\ \Phi_{i j}=\sigma_{i} \sigma_{j}+K \omega_{i} \omega_{j} & \text { (Gauss equation) } \\ D \sigma_{i}=0 & \text { (Codazzi equation) }\end{cases}
$$

Received February 27, 1959. 
Proof. Suppose there exists an immersion $i: M \rightarrow \bar{M}$. Since $M$ is simply connected, there is a unit normal vector field on the immersed manifold, $N$ being a differentiable $\left(=C^{\infty}\right)$ map from $M$ to the tangent of $\bar{M}$. Then the formula $\psi\left(m, f_{1}, \cdots, f_{a}\right)=\left(i(m), d i\left(f_{1}\right), \cdots, d i\left(f_{a}\right), N(m)\right)$ defines a differentiable map $\psi: F(M) \rightarrow F(\bar{M})$. (Denote by $\bar{R}_{a}, \bar{\varphi}, \ldots$ the structural elements of $\bar{M}$.) Note that $\psi \circ R_{a}=\bar{R}_{a} \circ \psi$ if $a \in O(d) \subset$ $O(d+1)$. This fact plus the uniqueness of the Riemannian connection of $M$ are used in the proof that

$$
\left\{\begin{aligned}
\omega_{i} & =\bar{\omega}_{i} \circ d \psi \\
0 & =\bar{\omega}_{d+1} \circ d \psi \\
\varphi_{i j} & =\bar{\varphi}_{i j} \circ d \psi .
\end{aligned}\right.
$$

Furthermore, the $R^{a}$-valued 1-form defined by (3) $\sigma_{i}=\varphi_{i, a+1} \circ \psi$ satisfies the conditions stated in the theorem. This form is, of course, one expression for the second fundamental form of the immersed manifold.

Conversely, given a form $\sigma$ on $F(M)$ with the stated properties we must produce an immersion $i: M \rightarrow \bar{M}$. To do this we first find a differentiable map $\varphi: F(M) \rightarrow F(\bar{M})$ satisfying the differential equations (2) and (3). Consider the 1-forms $\bar{\omega}_{i}-\omega_{i}, \bar{\omega}_{d+1}, \bar{\varphi}_{i j}-\phi_{i j}, \bar{\varphi}_{i, a+1}-\sigma_{i}$ on $F(M) \times F(\bar{M})$, where we use the same notation for a form on one factor and that form pulled back to the product manifold by a projection. We want to apply the Frobenius theorem to these forms. Taking account of the structural equations one sees that its hypothesis holds provided $\Sigma_{k} \sigma_{k} \omega_{k}=0 ; d \varphi_{i j}=-\Sigma_{k} \varphi_{i k} \varphi_{k j}+\sigma_{i} \sigma_{j}+K \omega_{i} \omega_{j}$; and $d \sigma_{i}=-\Sigma_{k} \varphi_{i k} \sigma_{k}$. But these conditions follow from the corresponding equations in (1)-in the case of the last one because for $\sigma$ (or any other $R^{a}$-valued horizontal equivariant 1-form on $F(M)$ we have $d \sigma_{i}=-\Sigma \varphi_{i k} \sigma_{k}+D \sigma_{i}$. Then if $(g, \bar{g}) \in F(M) \times F(\bar{M})$, an integral manifold through $(g, \bar{g})$ given by the Frobenius theorem is the graph of a differentiable function $\psi^{\prime}$ defined on a neighborhood $U$ of $g \in F(M)$, carrying $g$ to $\bar{g}$, and satisfying (2) and (3). Subject to these conditions $\phi^{\prime}$ is unique, except for the size of its domain. Further, one can show that $\mathscr{P}^{\prime}$ commutes with rightmultiplication in the sense that, where meaningful, $\phi^{\prime} \circ R_{a}$ and $\bar{R}_{a} \circ \varphi^{\prime}$ agree. This fact permits us to extend the local solution $\psi^{\prime}$ by rightmultiplication (in an obvious way) to a solution $\varphi: \pi^{-1}(V) \rightarrow F(\bar{M})$, where $V=\pi(U) \subset M$. Thus there exists a unique differentiable map $j: V \rightarrow \bar{M}$ such that $j \circ \pi=\bar{\pi} \circ \psi$ on $\pi^{-1}(V)$. We claim that $j$ is an immersion: In fact, suppose $f \in F(M)$ projects to $m \in V$, and let $\psi(f)=\bar{f} \in F(\bar{M})$. Now if $y \in F(M)_{f}$ projects to $x \in M_{m}$ we have

$$
\begin{aligned}
& \left\langle x, f_{i}\right\rangle=\omega_{i}(y)=\bar{\omega}_{i}(d \psi(y))=\left\langle d \pi(d \psi(y)), \bar{f}_{i}\right\rangle \\
& \quad=\left\langle d j(x), f_{i}\right\rangle \text {, and }\left\langle d j(x), f_{d+1}\right\rangle=\bar{\omega}_{d+1}(d \psi(x))=0 .
\end{aligned}
$$


This proves $j: V \rightarrow \bar{M}$ is an immersion; similarly one checks that its second fundamental form is $\sigma \mid \pi^{-1}(V)$. But an immersion is controlled by its second fundamental form; explicitly in the case at hand, if $j^{\prime}$ is another such immersion of $V$ in $\bar{M}$ with $j(m)=j^{\prime}(m)$ and $d j_{m}=d j_{m}^{\prime}$ for some one $m \in V$, then $j=j^{\prime}$. This uniqueness property, the simple connectedness of $M$, and the special character of $\bar{M}$ are the essential points in a proof (which we omit) that out of local immersions as above a global immersion $i: M \rightarrow \bar{M}$ can be constructed of which $\sigma$ is the second fundamental form.

2. The Gauss equation. Of the conditions (1) imposed on $\sigma$, the crucial one is the Gauss equation. Under the usual translation [1] of horizontal equivariant objects on $F(M)$ into objects on $M$, the curvature form becomes a function which to each $x, y \in M_{m}$ assigns a linear transformation $R_{x y}: M_{m} \rightarrow M_{m}$. Then then equation $\left\langle R_{x y}(u), v\right\rangle=\left\langle R_{m}(x y), u v\right\rangle$ defines the curvature transformation $R_{m}$ as a linear operator on the Grassmann space $\wedge^{2} M_{m}$. The function $m \rightarrow R_{m}$ is for our purposes the most convenient form of the curvature tensor $R$ of $M$. The form $\sigma$ translates to a function $S$ on $M$ with $S_{m}$ a linear operator on $M_{m}$, and the Gauss equation becomes $R=S \wedge S+K$, where $K$ denotes scalar multiplication by the constant curvature $K$ of $\bar{M}$.

Reversing the process, suppose that $S$ is a differentiable field of linear operators on the tangent spaces of $M$ such that $R=S \wedge S+K$. Let $\sigma$ be the horizontal, equivariant $R^{a}$-valued 1-form on $F(M)$ corresponding to $S$. Then $\Phi_{i j}=\sigma_{i} \sigma_{j}+K \omega_{i} \omega_{j}$. The other two conditions on $\sigma$ follow automatically if the rank of $R-K$, that is, the minimum rank of $R_{m}-K$ for $m \in M$, is not too small. Explicitly:

Lemma 1. (notation as above) Let $R=S \wedge S+K$. If $\operatorname{rank}(R-K) \geq$ 3 , then $\Sigma_{k} \sigma_{k} \omega_{k}=0$. If $\operatorname{rank}(R-K) \geq 4$, then $D \sigma_{i}=0$.

Proof. By a symmetry of $R$, shared by $K$, we have $\mathfrak{S}\langle S(x) S(u), y v\rangle=$ 0 , where $\mathfrak{S}$ denotes the sum over the cyclic permutations of $x, u, y$. Eliminating $v$ we get $\mathfrak{S}\{(\langle S(y), x\rangle-\langle y, S(x)\rangle) S(u)\}=0$. But since rank $S \wedge S \geq 3$, the same is true for $S$, and it follows that $\langle S(y), x\rangle=$ $\langle y, S(x)\rangle$. But the symmetry of $S$ is equivalent to $\Sigma_{k} \sigma_{k} \omega_{k}=0$.

To prove the second assertion (due essentially to T. Y. Thomas), we apply $D$ to the equation $\Phi_{i j}=\sigma_{i} \sigma_{j}+K \omega_{i} \omega_{j}$. Since $D \omega=0$ and $D \Phi=0$ (Bianchi identity) we get $D \sigma_{i} \wedge \sigma_{j}=\sigma_{i} \wedge D \sigma_{j}$. The rank condition implies rank $S \geq 4$, hence rank $\sigma \geq 4$. Thus the result is a consequence of the following.

LEMma 2. Let $x_{1}, \cdots, x_{a} \in V$, a finite-dimensional real vector space, and let $w_{1}, \cdots, w_{a} \varepsilon \wedge^{2} V$. If $x_{i} \wedge w_{j}=w_{i} \wedge x_{j}$ for all $1 \leq i, j \leq d$, 
and the vectors $x_{1}, \cdots, x_{a}$ span a subspace of dimension $\geq 4$, then $w_{1}=\cdots=w_{a}=0$.

Proof. We may suppose that $x_{1}, x_{2}, x_{3}, x_{4}$ are the first four elements of a basis $e_{1}, e_{2}, \cdots$ for $V$. Let $P=\{1,2,3,4\}$, and fix an index $p \in P$. By a standard Grassmann argument one can show that there is a $y_{p} \in V$ such that $w_{p}=y_{p} e_{p}$. Then $e_{p} \wedge w_{q}=w_{p} \wedge e_{q}$ implies $\left(y_{p}+y_{q}\right) e_{p} e_{q}=0$ for all $q \in P$. Thus $2 y_{p}=\left(y_{p}+y_{q}\right)+\left(y_{p}+y_{r}\right)-\left(y_{q}+y_{r}\right)$ is in the subspace spanned by $e_{p}, e_{q}, e_{r}$, where $q$ and $r$ are any elements of $P$ such that $p, q, r$ are all different. It follows that $y_{p}$ is a multiple of $e_{p}$, and thus $w_{p}=0$. But if $i>4$, then $e_{p} \wedge w_{i}=w_{p} \wedge e_{i}=0$ for all $p \in P$, so that $w_{i}=0$ also.

Summarizing, if $M$ and $\bar{M}$ are as in Theorem 1 and rank $(R-K) \geq$ 4 , then $M$ can be immersed in $\bar{M}$ if and only if $R-K$ is decomposable, i.e. expressible as $S \wedge S$ with $S$ a differentiable field of linear operators on the tangent spaces of $M$.

In the following section we consider the purely Grassmannian question of the decomposability of $R_{m}-K$ at a single point of $M$.

3. Decomposability. Let $V$ and $W$ be finite-dimensional real vector spaces, and let $T: \wedge^{2} V \rightarrow \wedge^{2} W$ be a linear transformation. To determine whether $T$ is decomposable we use the following definition: Three bivectors are crossed if any two, but not all three, are collinear, (a set of bivectors being called collinear if all have a common non-zero divisor, i.e. all are decomposable and the planes of the non-zero ones have a line in common.) One easily proves:

LEMMA 3. Bivectors $w_{1}, w_{2}, w_{3}$ are crossed if and only if there exist linearly independent vectors $x, y, z$ and non-zero numbers $K, L, M$ such that

$$
\left\{\begin{array}{l}
w_{1}=K x y \\
w_{2}=L x z \\
w_{3}=M y z
\end{array}\right.
$$

If $w_{1}, w_{2}, w_{3}$ are crossed, then in any expression (4) the sign of the product $K L M$ is always the same. (In fact, the vectors $x, y, z$ are unique up to non-zero scalar multiplication, so we need only check that changing the signs of any subset of $\{x, y, z\}$ does not change the sign of $K L M$.) In case $K L M>0$ we say that $w_{1}, w_{2}, w_{3}$ are coherently crossed. Note that if $T$ is decomposable then $T$ carries coherently crossed bivectors to bivectors which are either coherently crossed or coplanar. Our 
aim is to prove the converse when rank $T \geq 4$. (We do not need the easy cases of lower rank.)

Lemma 4. The following conditions on T are equivalent:

(a) $T$ carries decomposable bivectors to decomposable bivectors.

(b) T carries two collinear bivectors to two collinear bivectors.

(c) $T(x y) \wedge T(u v) \in \wedge^{2} W$ is skew-symmetric in its arguments.

Lemma 5. If rank $T \geq 4$ and $T$ carries crossed to crossed or coplanar bivectors, then $R$ carries collinear to collinear bivectors.

Proof. It is sufficient to prove collinearity is preserved in the case of three bivectors. Thus we must show that $T\left(e_{1} e_{2}\right), T\left(e_{1} e_{3}\right), T\left(e_{1} e_{4}\right)$ are collinear. Now any two of these bivectors are collinear, hence all three are either crossed or collinear. We assume the former and get a contradiction. If they are crossed there is a unique subspace $U$ of $W$, with dimension 3, such that the bivectors are in $\wedge^{2} U \subset \wedge^{2} W$. We may also assume that $e_{1}, e_{2}, e_{3}, e_{4}$ are linearly independent for otherwise we can reduce to the case of two collinear bivectors. Thus these vectors are part of a basis for $V$.

Case I. There is an index $i$ such that $T\left(e_{1} e_{i}\right) \notin \wedge^{2} U$. Consider $T\left(e_{1} e_{2}\right), T\left(e_{1} e_{3}\right), T\left(e_{1}\left(e_{4}+\delta e_{i}\right)\right)$, where $\delta$ is an arbitrarily small non-zero number. Now the last of these three bivectors is not in $\Lambda^{2} U$, while the union of the planes of the first two spans $U$. Hence all three are not in the second Grassmann product of any 3-dimensional subspace of $W$. Thus they are not crossed. On the other hand, any two are collinear, so all three are collinear. But this is a contradiction, for an arbitrarily samll change in the crossed bivectors $T\left(e_{1} e_{2}\right), T\left(e_{1} e_{3}\right)$, $T\left(e_{1} e_{4}\right)$ cannot produce collinear bivectors.

Case II. For all $i, T\left(e_{1} e_{i}\right) \in \mathcal{A}^{2} U$.

We prove the contradiction rank $T \leq 3$ by showing that $T\left(e_{p} e_{q}\right) \in \Lambda^{2} U$ for all $p, q$. If $T\left(e_{1} e_{p}\right)$ and $T\left(e_{1} e_{q}\right)$ are independent, then by hypothesis, $T\left(e_{p} e_{q}\right)$ is crossed with these two bivectors, hence is in $\Lambda^{2} U$. If they are dependent and $T\left(e_{1} e_{p}\right) \neq 0$, then by hypothesis $T\left(e_{1} e_{p}\right)$ and $T\left(e_{p} e_{q}\right)$ are coplanar and $T\left(e_{p} e_{q}\right) \in \Lambda^{2} U$. Finally, if $T\left(e_{1} e_{p}\right)=0$, then by Lemma 5 $0=T\left(e_{1} e_{p}\right) \wedge T\left(e_{r} e_{q}\right)=T\left(e_{1} e_{r}\right) \wedge T\left(e_{p} e_{q}\right)$ for $r=2,3,4$. But since $T\left(e_{1} e_{2}\right)$, $T\left(e_{1} e_{3}\right), T\left(e_{1} e_{4}\right)$ are crossed one easily deduces from these equations that $T\left(e_{p} e_{q}\right) \in \Lambda^{2} U$.

THeorem 2. Let $T: \wedge^{2} V \rightarrow \wedge^{2} W$ be a linear transformation of rank $\geq 4$. Then there exists a linear transformation $S: V \rightarrow W$ such that $T=S \wedge S$ if and only if $T$ carries coherently crossed to coherently 
crossed or coplanar bivectors.

Proof. We may choose a basis $e_{1}, \cdots, e_{d}$ for $V$ such that $T$ is never zero on the corresponding canonical basis for $A^{2} V$. Fix an index $1 \leq i \leq d$. By the preceding lemma there is a non-zero vector $u_{i} \in W$ such that $u_{i}$ divides each $T\left(e_{i} e_{j}\right), j=1, \cdots, d$. Furthermore this vector is unique up to scalar multiplication. To see this we need only show that these bivectors $T\left(e_{i} e_{j}\right)$ are not all coplanar. But if they were, then $T\left(e_{i} e_{j}\right), T\left(e_{i} e_{k}\right), T\left(e_{j} e_{k}\right)$, since not crossed, would have to be coplanar for all $j, k$, implying $\operatorname{rank} T \leq 1$.

Now let $i, j$ be different indices. We claim that $T\left(e_{i} e_{j}\right)=K_{i j} u_{i} u_{j}$, In fact, since there is an index $k$ such that the bivectors $T\left(e_{i} e_{j}\right)$ and $T\left(e_{i} e_{k}\right)$ are not coplanar, they are crossed with $T\left(e_{j} e_{k}\right)$. By Lemma 3 and the divisibility properties of $u_{i}, u_{j}, u_{k}$, it follows that these crossed bivectors may be written as $K u_{i} u_{j}, L u_{i} u_{k}, M u_{j} u_{k}$ respectively.

By changing the signs of $u_{2}, \cdots, u_{d}$ where necessary, we shall now arrange to have the number $K_{i j}(i<j)$ all positive. We can certainly get all $K_{i j}>0$ in this way. Consider $T\left(e_{1} e_{i}\right), T\left(e_{1} e_{j}\right), T\left(e_{i} e_{j}\right)$. If the first two bivectors are not coplanar, then all three are coherently crossed, hence the product $K_{1 i} K_{1 j} K_{i j}$, and consequently $K_{i j}$, are positive. If $T\left(e_{1} e_{i}\right)$ and $T\left(e_{1} e_{j}\right)$ are coplanar, we argue as follows: Since rank $T>1$ there is an index $k$ (say $k>j$ ) such that $u_{k}$ is not in the plane spanned by $u_{1}, u_{i}, u_{j}$. Thus $T\left(e_{1} e_{i}\right)$ and $T\left(e_{1} e_{k}\right)$ are not coplanar, so $K_{i k}>0$. Similarly $K_{j k}>0$. And since $u_{i}, u_{j}, u_{k}$ are independent, it follows that $K_{i j}>0$.

To complete the proof it will suffice to find numbers $\lambda_{1}, \cdots, \lambda_{d}$ such that for any $i<j$ we have $K_{i j}=\lambda_{i} \lambda_{j}$. For then the equation $T\left(e_{i} e_{j}\right)=$ $K_{i j} u_{i} u_{\text {, becomes }} T\left(e_{i} e_{j}\right)=\left(\lambda_{i} u_{i}\right)\left(\lambda_{j} u_{j}\right)$, and by definding $S: V \rightarrow W$ to be the linear transformation such that $S\left(e_{i}\right)=\lambda_{i} u_{i}$ we get $T=S \wedge S$.

Call a set $i, j, k$ of indices a triple if $i<j<k$ and $u_{i}, u_{j}, u_{k}$ are independent. For each triple consider the equations $K_{i j}=\lambda_{i} \lambda_{j}, K_{i j}=$ $\lambda_{i} \lambda_{k}, K_{j k}=\lambda_{j} \lambda_{k}$. Since the $K$ 's are positive there is a unique positive solution $\lambda_{i}, \lambda_{j}, \lambda_{k}$. Since each index $i$ is in at least one triple we get at least one such value for $\lambda_{i}$. We must show that the values obtained from two different triples containing $i$ are the same. We need only consider triples of the form $i, j, p$ and $i, j, q$, for it will be clear from the proof in this case that the position of $i$ in a triple is immaterial and that the case where five indices are involved may be reduced to the present one using rank $T \geq 4$. We know that

$$
\begin{array}{ll}
T\left(e_{i} e_{j}\right)=\lambda_{i} \lambda_{j} u_{i} u_{j} & T\left(e_{i} e_{j}\right)=\mu_{i} \mu_{j} u_{i} u_{j} \\
T\left(e_{i} e_{p}\right)=\lambda_{i} \lambda_{p} u_{i} u_{p} & T\left(e_{i} e_{q}\right)=\mu_{i} \mu_{q} u_{i} u_{q} \\
T\left(e_{j} e_{p}\right)=\lambda_{j} \lambda_{p} u_{j} u_{p} & T\left(e_{j} e_{q}\right)=\mu_{j} \mu_{q} u_{j} u_{q} .
\end{array}
$$


First consider the case in which the vectors $u_{i}, u_{j}, u_{p}, u_{q}$ are linearly independent. By Lemma 4, $T\left(e_{i} e_{p}\right) \wedge T\left(e_{j} e_{q}\right)=-T\left(e_{j} e_{p}\right) \wedge T\left(e_{i} e_{q}\right)$, but since $u_{i} u_{p} u_{j} u_{q} \neq 0$ this implies $\lambda_{i} \mu_{j}=\mu_{i} \lambda_{j}$. But also $\lambda_{i} \lambda_{j}=\mu_{i} \mu_{j}$, and since the numbers in the last two equations are all positive we get $\lambda_{i}=\mu_{i}$. Now suppose $u_{i}, u_{j} u_{p}, u_{q}$ are dependent, hence span a 3-dimensional subspace. Since rank $T \geq 4$ there must exist an index $r$ (say $r>p, q)$ such that $u_{i}, u_{j}, u_{p}, u_{r}$ and $u_{i} u_{j} u_{q}, u_{r}$ are each linearly independent. Thus the values of $\lambda_{i}$ determined by $i, j, p$ and $i, j, p$ are the same as that determined by $i, j, r$.

This shows the existence of $S$ such that $T=S \wedge S$; uniqueness up to sign is implicit in the proof, for the only ultimate element of choice is in the orientation of $u_{1}$, i.e. the use of $u_{1}$ rather than $-u_{1}$.

4. Coordinate criteria for decomposability. With notation as in the preceding section, fix bases $e_{1}, \cdots, e_{a}$ for $V$ and $f_{1}, \cdots, f_{\bar{a}}$ for $W$. Let $T_{i j}=T\left(e_{i} e_{j}\right)=\Sigma_{\alpha<\beta} T_{i j \alpha \beta} f_{\alpha} f_{\beta}$. What conditions on $T_{i j}$ are necessary and sufficient for $T$ to be decomposable, or alternatively (if rank $T \geq 4$ ) for $T$ to carry coherently crossed to coherently crossed or coplanar bivectors? Necessary is that $T$ carry decomposable to decomposable bivectors, and this is easily proved equivalent to

$$
T_{i j} \wedge T_{k l}=T_{k j} \wedge T_{i l} \text { for all } 1 \leq i, j, k, l \leq d
$$

This condition as well as the condition rank $T \geq 4$ are standardly expressible in terms of polynomials in $T_{i j \alpha \beta}$.

Lemma 6. Suppose that any two of the bivectors $a, b, c \in \wedge^{2} W$ are collinear, and let $a=\Sigma_{\alpha<\beta} A_{\alpha \beta} f_{\alpha} f_{\beta}$, similar expressions for $b, c$. Then $a, b, c$ are coherently crossed if and only if there exist indices $1 \leq \alpha<$ $\beta<\gamma \leq \bar{d}$ such that

$$
\Delta(\alpha \beta \gamma)=\left|\begin{array}{lll}
A_{\alpha \beta} & A_{\alpha \gamma} & A_{\beta \gamma} \\
B_{\alpha \beta} & B_{\alpha \gamma} & B_{\beta \gamma} \\
C_{\alpha \beta} & C_{\alpha \gamma} & C_{\beta \gamma}
\end{array}\right|>0
$$

Proof. The bivectors $a, b, c$ are either crossed or collinear. We show:

(1) if crossed, then for some $\alpha, \beta, \gamma$ we have $\Delta(\alpha \beta \gamma) \neq 0$,

(2) if coherently crossed, then each such non-zero determinant is positive,

(3) if collinear, then each such determinant is zero.

For $\alpha, \beta, \gamma$ let $x \rightarrow \bar{x}$ be the natural projection of $W$ onto the subspace $U$ spanned by $f_{\alpha}, f_{\beta}, f_{\gamma}$; same notation for the induced projection of $\wedge^{2} W$ onto $\wedge^{2} U$. In the first two cases above we can write $a, b, c$ in 
the form of (4), hence $\bar{a}=K \bar{x} \bar{y}, \bar{b}=L \bar{x} \bar{z}, \bar{c}=M \bar{y} \bar{z}$. For (1), since $x, y, z$ independent there are indices $\alpha, \beta, \gamma$ such that $\bar{x}, \bar{y}, \bar{z}$ are independent, hence $\bar{a}, \bar{b}, \bar{c}$ are independent, and the result follows. For (2), suppose $\Delta(\alpha \beta \gamma) \neq 0$. Using the above notation we have $K L M>0$. Notice that any two canonical bases (lexicographic order) for $\wedge^{2} U$ have the same orientation. Thus $\Delta(\alpha \beta \gamma)>0$. The proof of (3) is similar.

A further necessary condition for decomposability of $T$ is that $T_{i j}$, $T_{i k}, T_{j k}$ be coherently crossed or coplanar. Assuming (1), this is equivalent to

(6) If $1 \leq i<j<k \leq d$, then either $T_{i j}, T_{i k}, T_{j k}$ are coplanar or there exist indices $1 \leq \alpha<\beta<\gamma \leq \bar{d}$ such that

$$
\left|\begin{array}{lll}
T_{i j \alpha \beta} & T_{i j \alpha \gamma} & T_{i j \beta \gamma} \\
T_{i k \alpha \beta} & T_{i k \alpha \gamma} & T_{i k \beta \gamma} \\
T_{i k \alpha \beta} & T_{j k \alpha \gamma} & T_{j k \beta \gamma}
\end{array}\right|>0 .
$$

If the basis $e_{1}, \cdots, e_{a}$ is such that all $T_{i j} \neq 0$, then (5) and (6) are necessary and sufficient for the decomposability of $T$, for Lemma 5 and Theorem 2 use no more than this. For an arbitrary basis, however, they are not enough, as one can see from simple examples. We must add, say

(7) If $T_{i j}=T_{i k}=0$, then either $T_{j k}=0$, or, for all $r, T_{i r}=0$.

Now one can prove the following lemma by reducing to the case in which all $T_{i j} \neq 0$.

LEMMA 7. Let $T: \wedge^{2} V \rightarrow \wedge^{2} W$ be a linear transformation with rank $T \geq 4$. Then $T$ is decomposable if and only if, relative to arbitrary canonical bases for $\wedge^{2} V$ and $\wedge^{2} W$, conditions (5), (6), (7) hold.

5. Summary. Again let $R$ be the curvature transformation of the simply connected manifold $M$. For simplicity we discuss the case $\bar{M}=R^{a+1}$. Assume that (at each point) rank $R \geq 4$ and $R$ carries coherently crossed to coherently crossed or coplanar bivectors. It is clear that the proof of Theorem 2 applies simultaneously to all $R_{n}$ with $n$ any point of a convex neighborhood $C$ of $m \in M$. One need only use the simple connectedness of $C$ to choose the orientations of the various choices of $u_{1}$ consistently. We thus obtain a differentiable field of linear operators $S$ such that $R=S \wedge S$, first locally, then as usual, globally. When rank $R \geq 3$ we can still prove $R$ decomposable, but the Codazzi equation may fail; thus our criterion for immersion, while always sufficient, is necessary only in the case of immersions for which the second fundamental form $S$ has rank at least four. Call such an immersion 4-regular. 
This same argument, with $R-K$ in place of $K$, proves

THEOREM 3. Let $M$ be a simply connected d-dimensional manifold $(d \geq 4)$ with curvature transformation $R$. Let $\bar{M}(K)$ be a complete $(d+1)$-dimensional manifold of constant curvature $K$. Then $M$ has a 4-regular immersion in $\bar{M}(K)$ if and only if rank $(R-K) \geq 4$ and $R-K$ carries coherently crossed to coherently crossed or coplanar bivectors, i.e. conditions (5), (6), (7) hold at eaeh point of $M$.

For a given $M$ one may ask for the set $\mathscr{K}$ of numbers $K$ such that $M$ has a regular immersion in an $\bar{M}(K)$. Consider two cases:

(i) If $R$ does not preserve decomposability, say $R(x y)^{2} \neq 0$, then $M$ is not immersible in $R^{l+1}$ and $\mathscr{K}$ contains at most the number $K$ determined by the necessary condition $R(x y)=S(x) S(y)+K x y$. We check as above whether $K \in \mathscr{K}$.

(ii) If $R$ preserves decomposability, so that (5) holds, $\mathscr{K}$ may well be infinite. By studying conditions (6), (7) one can characterize $\mathscr{K}$ in terms of polynomials in an unknown $K$ and the coordinates of $R$.

\section{REFERENCES}

1. W. Ambrose and I.M. Singer, On homogeneous Riemannian manifolds, Duke Math. J., 25 (1958), 647-669.

2. T. Y. Thomas, Riemannian spaces of class one and their charactetization, Acta Math., 67 (1936), 169-211.

Massachusetts Institute of TeChNology, UNIVERSITY OF CALIFORNIA, LOS ANGELES 



\section{PACIFIC JOURNAL OF MATHEMATICS}

EDITORS

David Gilbarg

Stanford University

Stanford, California

R. A. Beaumont

University of Washington

Seattle 5 , Washington
A. L. Whiteman

University of Southern California

Los Angeles 7, California

L. J. PAIGE

University of California

Los Angeles 24, California

\section{ASSOCIATE EDITORS}

\author{
E. F. BECKENBACH \\ C. E. BURGESS \\ E. HEWITT \\ A. HORN
}

\author{
V. GANAPATHY IYER \\ R. D. JAMES \\ M. S. KNEBELMAN \\ L. NACHBIN
}
I. NIVEN
E. G. STRAUS
T. G. OSTROM
G. SZEKERES
H. L. ROYDEN
F. WOLF
M. M. SCHIFFER
K. YOSIDA

\section{SUPPORTING INSTITUTIONS}

\author{
UNIVERSITY OF BRITISH COLUMBIA \\ CALIFORNIA INSTITUTE OF TECHNOLOGY \\ UNIVERSITY OF CALIFORNIA \\ MONTANA STATE UNIVERSITY \\ UNIVERSITY OF NEVADA \\ OREGON STATE COLLEGE \\ UNIVERSITY OF OREGON \\ OSAKA UNIVERSITY \\ UNIVERSITY OF SOUTHERN CALIFORNIA
}

\author{
STANFORD UNIVERSITY \\ UNIVERSITY OF TOKYO \\ UNIVERSITY OF UTAH \\ WASHINGTON STATE COLLEGE \\ UNIVERSITY OF WASHINGTON \\ $* \quad * \quad *$ \\ AMERICAN MATHEMATICAL SOCIETY \\ CALIFORNIA RESEARCH CORPORATION \\ HUGHES AIRCRAFT COMPANY \\ SPACE TECHNOLOGY LABORATORIES
}

Mathematical papers intended for publication in the Pacific Journal of Mathematics should be typewritten (double spaced), and the author should keep a complete copy. Manuscripts may be sent to any one of the four editors. All other communications to the editors should be addressed to the managing editor, L. J. Paige at the University of California, Los Angeles 24, California.

50 reprints per author of each article are furnished free of charge; additional copies may be obtained at cost in multiples of 50 .

The Pacific Journal of Mathematics is published quarterly, in March, June, September, and December. The price per volume (4 numbers) is $\$ 12.00$; single issues, $\$ 3.50$. Back numbers are available. Special price to individual faculty members of supporting institutions and to individual members of the American Mathematical Society: $\$ 4.00$ per volume; single issues, $\$ 1.25$.

Subscriptions, orders for back numbers, and changes of address should be sent to Pacific Journal of Mathematics, 2120 Oxford Street, Berkeley 4, California.

Printed at Kokusai Bunken Insatsusha (International Academic Printing Co., Ltd.), No. 6, 2-chome, Fujimi-cho, Chiyoda-ku, Tokyo, Japan.

PUBLISHED BY PACIFIC JOURNAL OF MATHEMATICS, A NON-PROFIT CORPORATION

The Supporting Institutions listed above contribute to the cost of publication of this Journal, but they are not owners or publishers and have no responsibility for its content or policies. 


\section{Pacific Journal of Mathematics}

\section{Vol. 9, No. 4 \\ August, 1959}

Frank Herbert Brownell, III, A note on Kato's uniqueness criterion for

Schrödinger operator self-adjoint extensions ............... 953

Edmond Darrell Cashwell and C. J. Everett, The ring of number-theoretic

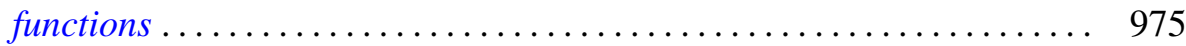

Heinz Otto Cordes, On continuation of boundary values for partial

differential operators ............................. 987

Philip C. Curtis, Jr., n-parameter families and best approximation . . . . . . 1013

Uri Fixman, Problems in spectral operators . . . . . . . . . . . . . . . 1029

I. S. Gál, Uniformizable spaces with a unique structure .............. 1053

John Mitchell Gary, Higher dimensional cyclic elements ............ 1061

Richard P. Gosselin, On Diophantine approximation and trigonometric

polynomials ..................................... 1071

Gilbert Helmberg, Generating sets of elements in compact groups ........ 1083

Daniel R. Hughes and John Griggs Thompson, The H-problem and the

structure of $H$-groups .................................. 1097

James Patrick Jans, Projective injective modules ................. 1103

Samuel Karlin and James L. McGregor, Coincidence properties of birth and

death processes ..................................... 1109

Samuel Karlin and James L. McGregor, Coincidence probabilities ........ 1141

J. L. Kelley, Measures on Boolean algebras ................... 1165

John G. Kemeny, Generalized random variables ................... 1179

Donald G. Malm, Concerning the cohomology ring of a sphere bundle ... . . 1191

Marvin David Marcus and Benjamin Nelson Moyls, Transformations on

tensor product spaces .................................. 1215

Charles Alan McCarthy, The nilpotent part of a spectral operator ........ 1223

Kotaro Oikawa, On a criterion for the weakness of an ideal boundary

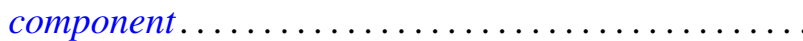

Barrett O'Neill, An algebraic criterion for immersion . . ............... 1239

Murray Harold Protter, Vibration of a nonhomogeneous membrane ... . . . . 1249

Victor Lenard Shapiro, Intrinsic operators in three-space . . . . . . . . . . . 1257

Morgan Ward, Tests for primality based on Sylvester's cyclotomic

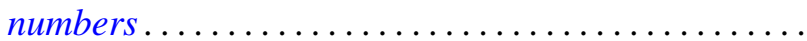

L. E. Ward, A fixed point theorem for chained spaces ....

Alfred B. Willcox, Šilov type $C$ algebras over a connected locally compact

abelian group..................................... 1279

Jacob Feldman, Correction to "Equivalence and perpendicularity of

Gaussian processes" ........................ 